\title{
Global Cancer Inequalities
}

\author{
Miranda M. Fidler* and Freddie Bray \\ Section of Cancer Surveillance, International Agency for Research on Cancer, Lyon, France
}

Social inequalities in cancer are increasingly relevant to research, implementation science, and policy. In this brief perspective we provide an overview of global cancer inequalities by assessing different outcomes according to the Human Development Index (HDI); the HDI is a United Nations Development Programme composite indicator including the following measures: (i) access to education (based on mean and expected years of schooling), (ii) a long and healthy life (based on life expectancy), and (iii) a decent standard of living (based on gross national income per capita). We additionally touch upon the importance of prevention, access to oncological services, and the need to monitor progress in reducing and avoiding inequalities at subnational, national, world region, and global levels.

Keywords: social inequalities, cancer, global, incidence, mortality, DALYs

\section{OPEN ACCESS}

Edited by:

Friederike Erdmann Danish Cancer Society, Denmark

Reviewed by:

Bárbara Peleteiro,

Universidade do Porto, Portugal

Alireza Sadjadi,

Tehran University of Medical Sciences,

Kjetil Søreide

University of Bergen, Norway

*Correspondence:

Miranda M. Fidler

fidlerm@fellows.iarc.ff

Specialty section:

This article was submitted to

Cancer Epidemiology and Prevention,

a section of the journal

Frontiers in Oncology

Received: 30 May 2018

Accepted: 13 July 2018

Published: 14 August 2018

Citation:

Fidler MM and Bray F (2018) Global

Cancer Inequalities.

Front. Oncol. 8:293.

doi: 10.3389/fonc.2018.00293
Although social inequalities are well documented in cancer at macro and micro levels (1), their strong and persistent presence across the cancer continuum is increasingly relevant to research, implementation science, and policy. Cancer disparities research has moved beyond macroeconomic analyses involving comparative assessments of socioeconomic status or national income to investigate the multiple determinants of social inequality, exemplified through the assessment of demographic factors, including gender, age, race and ethnicity, and indigenous status. The enquiry as to how the global cancer burden (incidence, mortality, survival, disability-adjusted life years) is impacted by inequalities in socioeconomic development has moved on too. The exploration of the scale and profile of cancer, once set against the time-honored dichotomy of populations being "developing" or "developed," has been superseded by the four-tier Human Development Index (HDI) (2), a broad marker of inequality and cancer transition between countries $(3,4)$.

The HDI asserts that an assessment of development is not just about economic growth but rather how national policies impact on human choices. The indicator is a composite measure incorporating (i) access to education (based on mean and expected years of schooling), (ii) a long and healthy life (based on life expectancy), and (iii) a decent standard of living (based on gross national income per capita) (2). It is commonly presented, as it is here, according to United Nations Development Programme's pre-defined cut-points representing four tiers of the HDI (from low HDI, e.g., nations with lowest human development values, through to medium, high, and very high, e.g., those with highest human development values).

Using this criterion, a strong correlation between the magnitude of the overall cancer incidence and the corresponding HDI level can be observed, with the overall cancer rates broadly increasing with level of human development (4). When assessed by specific cancers, positive relationships between the HDI level and incidence rate in 2012 were observed in both sexes for the following cancers: brain/nervous system, colorectum, gallbladder, kidney, leukemia, lung, multiple myeloma, pancreas, and thyroid; a positive relationship was also observed for bladder, lip/oral cavity, other pharyngeal, and testicular cancers, Hodgkin lymphoma, and melanoma in males, and breast, corpus uteri, and ovarian cancers and non-Hodgkin lymphoma in females (4). A negative association 


\section{Low HDI}

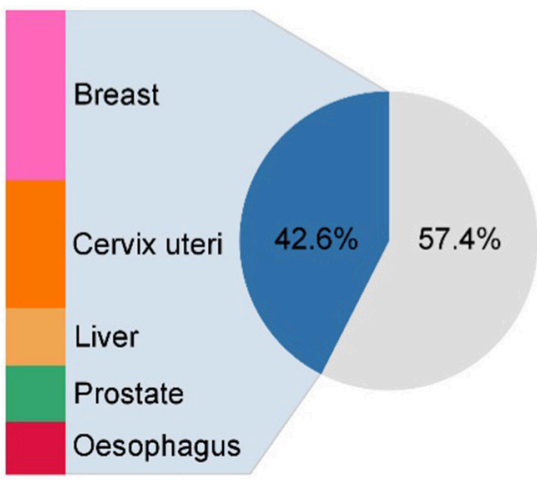

\section{0,000 new cases}

\section{High and Very High HDI}

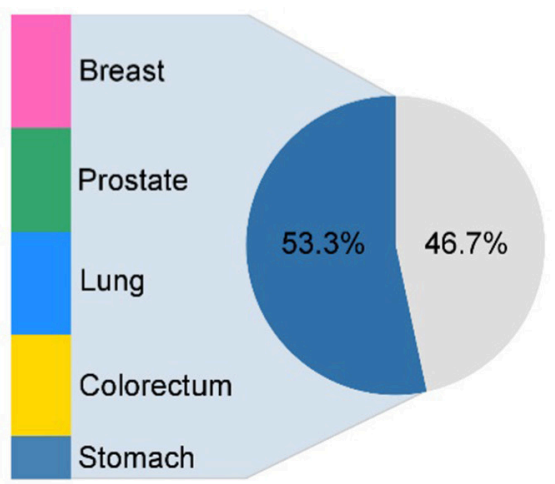

\section{9 million new cases}

FIGURE 1 | Number of new incidence cases and proportion of top five cancers in for the low Human Development Index (HDI) and high/very high HDI level as estimated in GLOBOCAN 2012.

between the HDI level and incidence rate was observed for cervical and other pharyngeal cancers and Kaposi sarcoma in females (4).

In terms of cancer profiles, the distribution of common cancers is quite different by HDI level, with infection- and poverty-related cancers (e.g., cervical and liver cancer) still dominating in low HDI nations, in contrast to high and very high HDI countries, where prostate, breast, colorectal, and lung are the major cancers (Figure 1). An increased burden of infectionrelated cancers with lower HDI is highlighted when one examines the population fractions of cancers attributable to infectious agents, which were estimated to be $25,22,13$, and $8 \%$ in low, medium, high, and very high HDI countries, respectively, in 2012 (5).

Other inequalities in the global cancer burden have also been noted when assessed by the HDI, including mortality (6), disability-adjusted life years (7), and relative gains in life expectancy (8). An important additional marker of cancer inequality pertains to HDI stratifications of the future cancer incidence burden, which reveals that the number of new cancer cases in future years will be proportionally greatest in lower HDI settings (Figure 2), with low and medium HDI countries projected to see a 112 and $86 \%$ respective increase in their incidence burden from 2012 to 2035 . Hence the nations currently least equipped to deal with a pending increase in the number of cancer patients year-on-year will be most impacted.

Such inequalities can only be expected to widen unless resource-dependant, effective, and cost-effective interventions are urgently implemented $(9,10)$. In particular, vaccination will be a key preventive strategy in low HDI settings given the high burden of infection-related cancers (5). Among the most important infections associated with cancer are hepatitis B virus and human papillomavirus, both of which have highly effective vaccines to prevent liver cancer and cervical, anal, vulvar, vaginal, and penial cancers, respectively (11). Tobacco control is another main priority for cancer control in transitioning countries given the number of smokers is projected to increase in these countries $(12,13)$. Finally, as social and economic transition increases the prevalence of sedentary jobs, urban living, and high caloric intake, an opportunity for prevention exists for less developed countries to avoid known adverse lifestyle risk factors like obesity, low physical activity, and higher alcohol intake, which cause many of the cancers commonly seen in the most developed countries.

With a growing cancer burden, access to appropriate, affordable, and equitable treatment will also be crucial in lower HDI settings, especially as the current availability of essential cancer medicines (14), cancer surgery (15), and radiotherapy facilities (16) is sparse. Preventing exposures to risk factors, early detection, effective treatment, and palliative care requires support and resources, however. More broadly, governments from around the world adopted a Cancer Resolution at the World Health Assembly in 2017, building on the United Nations Sustainable Development Goals 2030 (SDG), and the SDG 3.4 target of a reduction of the premature mortality from noncommunicable diseases by one-third by 2030 . Prevention and early detection are given prominence in the Resolution, with an emphasis on tobacco control policies within the World Health Organization Framework Convention on Tobacco Control, as well as affordable and feasible vaccine and screening programs. Furthermore, measuring the cancer burden to inform planning, through the development of population-based cancer registries, is also given central importance.

In summary, the HDI provides a useful framework to map out the continuing transitions in cancer globally, and highlights the clear reality of increasing inequalities in countries 


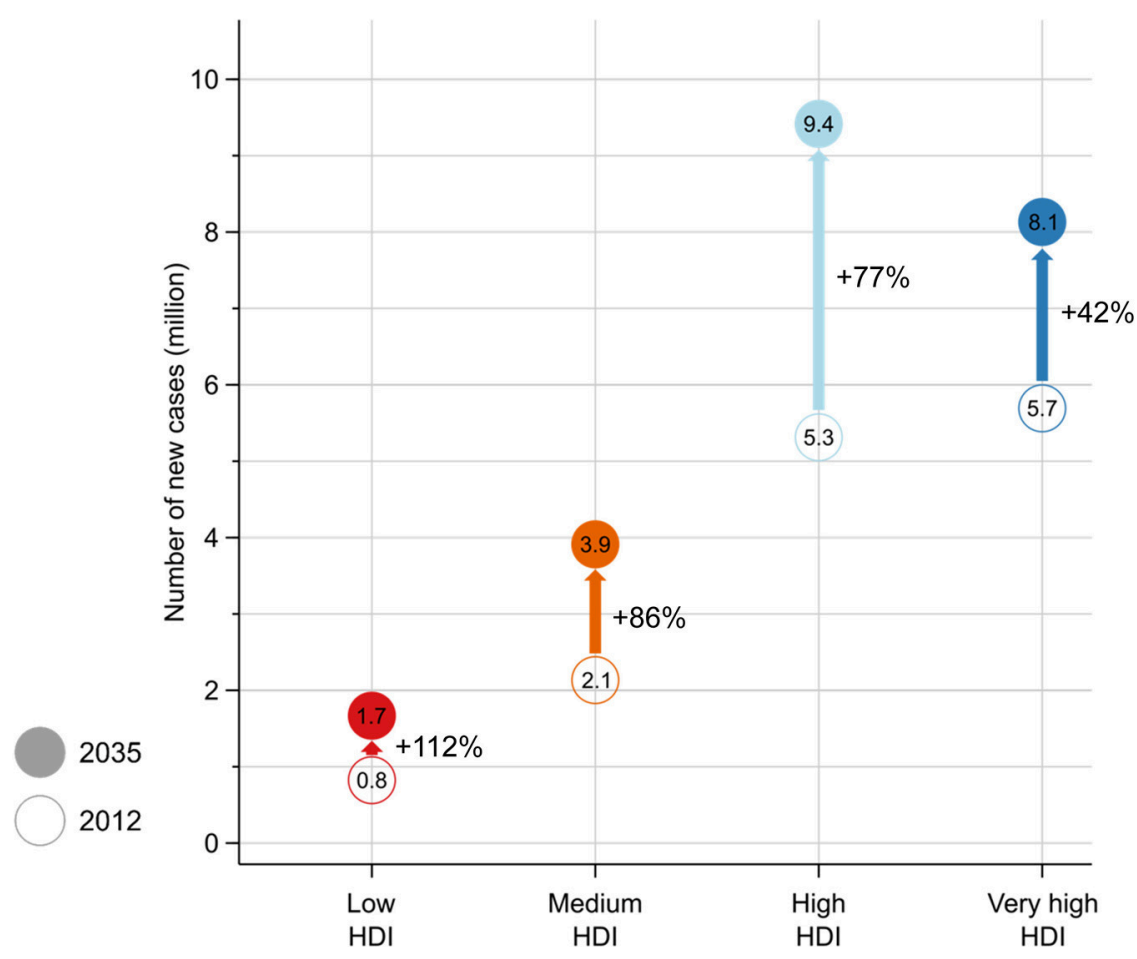

FIGURE 2 | Number of estimated new cancer cases in 2012 and 2035 by the four-tier Human Development Index (HDI), with the proportional increase (\%) in the number of cases indicated.

presently indexed at lower levels of the HDI. That said, there remains a need for an integration of social indicators in cancer research across the continuum, as well as the use of innovative methodologies, in order to monitor progress in reducing and avoiding inequalities at subnational, national, world region, and global levels. This is exemplified by the fact that governments have acknowledged the presence of social inequalities in cancer in the Cancer Resolution, which notes that “...certain population groups experience inequalities in risk factor exposure and in access to screening, early diagnosis and timely and appropriate treatment, and that they also experience poorer outcomes for cancer," and recommends including measurements of inequalities in the collection of high-quality population-based incidence and mortality data on cancer.

As cancer is emerging as the leading cause of premature death, given ongoing displacements of deaths from infection and parasitic diseases in the lower HDI spectrum and cardiovascular diseases in decline at the higher end (8), the development and implementation of operational cancer control plans that include feasible, affordable, and sustainable interventions is now imperative worldwide, and most markedly

\section{REFERENCES}

1. Kogevinas M, Pearce N, Susser M, Boffetta $\mathrm{P}$ editors. Social Inequalities and Cancer, IARC Scientific Publication No. 138. Lyon: International Agency for Research on Cancer (1997). Available in countries undergoing major social and economic transition. Such efforts can therefore be seen not only as an effort to reduce the widening gaps in cancer inequality, but also as a means to decrease inequalities across the spectrum of causes.

\section{DATA SOURCE AVAILABILITY}

GLOBOCAN 2012 data is freely available for download at http:// globocan.iarc.fr/Default.aspx. Human Development Index data is freely available for download at http://hdr.undp.org/en/data.

\section{AUTHOR CONTRIBUTIONS}

MF drafted the article, FB revised the article, MF and FB agreed to submission.

\section{ACKNOWLEDGMENTS}

The authors thank Mathieu Laversanne for undertaking the projection analysis and creating Figure 2.

online at: https://www.iarc.fr/en/publications/pdfs-online/epi/sp138/ SP138.pdf

2. United Nations Development Programme. Human Development Report 2015. (2015). p. 1-10. Available online at: http://hdr.undp.org/sites/default/files/ 2015_human_development_report_1.pdf [Accessed April 12, 2017]. 
3. Bray F, Jemal A, Grey N, Ferlay J, Forman D. Global cancer transitions according to the Human Development Index (2008-2030): a population-based study. Lancet Oncol. (2012) 13:790-801. doi: 10.1016/S1470-2045(12)70211-5

4. Fidler MM, Soerjomataram I, Bray F. A global view on cancer incidence and national levels of the human development index. Int J Cancer. (2016) 139:2436-46. doi: 10.1002/ijc.30382

5. Plummer M, de Martel C, Vignat J, Ferlay J, Bray F, Franceschi S, et al. Global burden of cancers attributable to infections in 2012: a synthetic analysis. Lancet Glob Heal. (2016) 4:e609-16. doi: 10.1016/S2214-109X(16)30143-7

6. Fidler MM, Bray F, Soerjomataram I. The global cancer burden and human development: a review. Scand J Public Health (2017) 46:27-36. doi: $10.1177 / 1403494817715400$

7. Soerjomataram I, Lortet-Tieulent J, Parkin D, Ferlay J, Mathers C, Forman D, et al. Global burden of cancer in 2008: a systematic analysis of disability-adjusted life-years in 12 world regions. Lancet (2012) 380:1840-50. doi: 10.1016/S0140-6736(12)60919-2

8. Cao B, Bray F, Beltrán-Sánchez H, Ginsburg O, Soneji S, Soerjomataram I. Benchmarking life expectancy and cancer mortality: global comparison with cardiovascular disease 1981-2010. BMJ (2017) 357:j2765. doi: 10.1136/bmj.j2765

9. World Health Organization. Seventieth World Health Assembly Update, 30 May 2017. (2017). Available online at: http://www.who.int/mediacentre/news/ releases/2017/vector-control-ncds-cancer/en/ [Accessed February 28, 2018].

10. World Health Organization. Updating Appendix 3 of the Global NCD Action Plan 2013-2020. (2017). Available online at: http://www.who.int/ncds/ governance/technical_annex.pdf

11. International Agency for Research on Cancer. World Cancer Report 2012 (2014).
12. Giovino GA, Mirza SA, Samet JM, Gupta PC, Jarvis MJ, Bhala N, et al. Tobacco use in 3 billion individuals from 16 countries: an analysis of nationally representative cross-sectional household surveys. Lancet (2012) 380:668-79. doi: 10.1016/S0140-6736(12)61085-X

13. Mathers CD, Loncar D. Projections of global mortality and burden of disease from 2002 to 2030. PLoS Med. (2006) 3:e442. doi: 10.1371/journal.pmed.0030442

14. WHO (2015). Medicines for Treatment of the Following Cancers-Review-EML and EMLc. Available online at: http://www.who. int/selection_medicines/committees/expert/20/applications/cancer/en/ [Accessed September 30, 2016].

15. Sullivan R, Alatise OI, Anderson BO, Audisio R, Autier P, Aggarwal A, et al. Global cancer surgery: delivering safe, affordable, and timely cancer surgery. Lancet Oncol. (2015) 16:1193-224. doi: 10.1016/S1470-2045(15)00223-5

16. Atun R, Jaffray DA, Barton MB, Bray F, Baumann M, Vikram B, et al. Expanding global access to radiotherapy. Lancet Oncol. (2015) 16:1153-86. doi: $10.1016 /$ S1470-2045(15)00222-3

Conflict of Interest Statement: The authors declare that the research was conducted in the absence of any commercial or financial relationships that could be construed as a potential conflict of interest.

Copyright (C) 2018 Fidler and Bray. This is an open-access article distributed under the terms of the Creative Commons Attribution License (CC BY). The use, distribution or reproduction in other forums is permitted, provided the original author(s) and the copyright owner(s) are credited and that the original publication in this journal is cited, in accordance with accepted academic practice. No use, distribution or reproduction is permitted which does not comply with these terms. 